\title{
Primary intradural extramedullary Ewing sarcoma: A case report and literature review
}

\author{
YUYA IZUBUCHI $^{1}$, HIDEAKI NAKAJIMA ${ }^{1}$, KAZUYA HONJOH $^{1}$, \\ YOSHIAKI IMAMURA $^{2}$, TAKAYUKI NOJIMA ${ }^{3}$ and AKIHIKO MATSUMINE ${ }^{1}$ \\ ${ }^{1}$ Department of Orthopaedics and Rehabilitation Medicine, University of Fukui Faculty of Medical Sciences; \\ ${ }^{2}$ Division of Diagnostic Pathology/Surgical Pathology, University of Fukui Hospital, Eiheiji, Fukui 910-1193; \\ ${ }^{3}$ Department of Pathology, Kanazawa University, Kanazawa, Ishikawa 920-8641, Japan
}

Received January 21, 2020; Accepted May 27, 2020

DOI: $10.3892 / \mathrm{ol} .2020 .11786$

\begin{abstract}
Tumors of the spinal cord and cauda equina show a wide spectrum of histology and require careful diagnosis and treatment. Primary intradural extramedullary Ewing sarcoma (IEES) is extremely rare, and initial imaging and clinical findings for this tumor mimic those of benign intradural spinal tumors. The present report describes a case of a 35 -year-old woman who presented with IEES with meningeal seeding, and the literature on the management and clinical course of these tumors was reviewed. An examination revealed right-side leg dominant rapidly progressive hypoesthesia and motor deficits. MRI identified intradural tumors at the T12-L1 and L4-5 levels. Growth and an increase in the number of intradural tumors occurred over 3 weeks. Gadolinium-contrast T1-weighted MRI revealed diffusely enhanced signals for tumorous lesions with meningeal seeding in the cervical and thoracic spinal cord. Excision of a tumor at T12-L1 was performed to alleviate the symptoms and to make a histologic diagnosis. Microscopically, the tumor consisted of dense sheets of small round cells. Immunohistochemically, tumor cells showed intense and diffuse positive staining for CD99, ETS transcription factor ERG and Fli-1 proto-oncogene, ETS transcription factor (FLI1). The sequence analysis revealed the EWS RNA binding protein 1-FLI1 fusion transcript. The lesion was diagnosed as primary intradural ES. Adjuvant chemotherapy following radiotherapy for the whole spine was performed; however, multiple brain metastases were found at 10 months after diagnosis and the patient died of diffusely disseminated disease limited to the central nervous system without evidence of distant metastases at 16 months after the
\end{abstract}

Correspondence to: Dr Hideaki Nakajima, Department of Orthopaedics and Rehabilitation Medicine, University of Fukui Faculty of Medical Sciences, 23-3 Matsuoka Shimoaizuki, Eiheiji, Fukui 910-1193, Japan

E-mail: nhideaki@u-fukui.ac.jp

Key words: Ewing sarcoma, intradural tumor, cauda equina tumor, radiotherapy, adjuvant chemotherapy, meningeal seeding initial diagnosis. In a literature review of IEES cases, the 1- and 5 -year overall survival rates were 79.8 and $26.6 \%$, respectively, and the 1-, 2- and 5-year progression-free survival rates were $61.0,52.3$ and $10.9 \%$, respectively. Therefore, primary IEES has a poor prognosis compared with ES of bone, and novel agents and treatment strategies are required.

\section{Introduction}

Tumors of the spinal cord and cauda equina have a wide spectrum of histology and require care with diagnosis and surgical intervention. The differential diagnosis for intradural spinal tumors includes meningioma, nerve sheath tumors such as schwannoma or neurofibroma, astrocytoma, ependymoma, and metastasis. Spinal cord and cauda equina tumors are uncommon neoplasms, and the majority is extramedullary tumors (1). Spinal involvement of extraskeletal Ewing sarcoma in the epidural space or paravertebral area is also a differential disease (2). Ewing sarcoma is an aggressive bone and soft tissue tumor that usually affects adolescents and young adults $(3,4)$. The Ewing sarcoma family of tumors are a group of high-grade small round cell tumors, including primitive neuroectodermal tumor (pPNET) and Askin tumor. Extraskeletal Ewing sarcoma is more likely to arise in axial locations, compared to Ewing sarcoma of bone (5). Most cases of Ewing sarcoma occur in the long bones, pelvis, or ribs, and rarely in extraskeletal regions such as the paravertebral or epidural space, whereas a primary intradural extramedurally Ewing sarcoma (IEES) is extremely rare. In general, Ewing sarcoma is treated with a multimodal approach including surgery and/or focal radiotherapy, in addition to systematic chemotherapy (4). However, because of its rarity and limited evidence regarding the therapeutic aspects of IEES, there are no standard treatment guidelines for these tumors even though the aggressive malignant tumor causes severe neurologic morbidity and mortality without appropriate treatment. In addition, the initial imaging and clinical findings of IEES mimic those for benign intradural spinal tumors. Therefore, it is important for oncologists and neurosurgeons to be familiar with the clinical presentation and evaluation of IEES. Here, we describe a case of IEES with meningeal seeding, and we present a literature review of the management and clinical course of this type of tumor. 

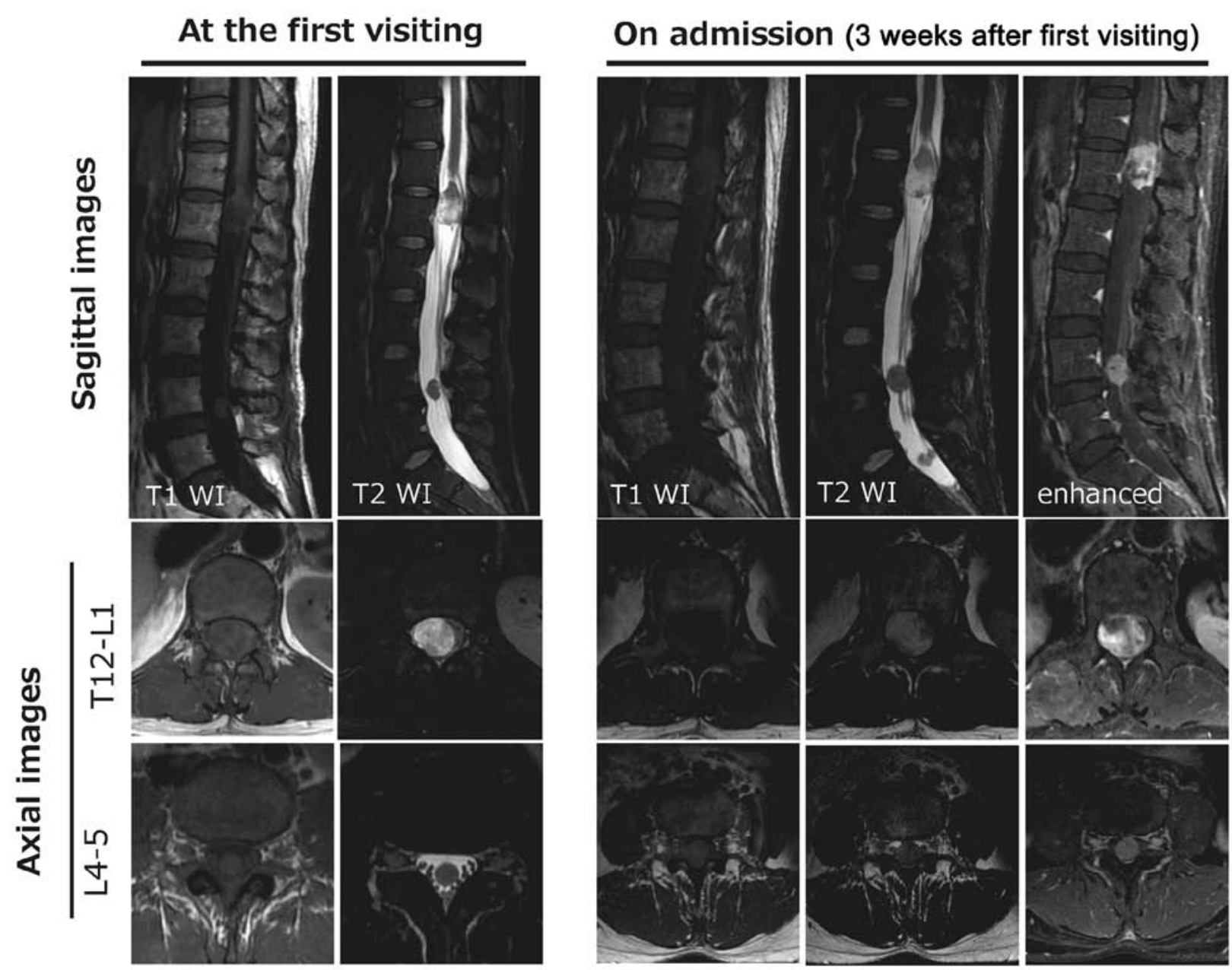

Figure 1. Sagittal and axial (T12-L1 and L4-5) thoracolumbar magnetic resonance images at the first visit and on admission (3 weeks after the first visit). Growth and increased numbers of semi-oval shaped lesions (isointense signal on T1-weighted, inhomogeneous hyperintense signal on T2-weighted and diffuse gadolinium-enhanced images) occurred within this period.

\section{Case report}

A previously healthy 35-year-old woman developed severe lumbago and radicular leg pain on both sides. The symptoms gradually worsened for 2 months after appearance and paresthesia of both legs progressed. Neurologically, straight leg raising and femoral nerve stretch tests were negative on both sides. There was hypoesthesia in the right-side dominant L5-S1 dermatomes. Patellar tendon and Achilles tendon reflexes were normal and Babinski and Chaddock reflexes were negative on both sides. The manual muscle test score was 3 in the right gastrocnemius muscle. Bladder and bowel functions were normal.

Magnetic resonance imaging (MRI) at a previous hospital showed tumorous lesions at the T12-L1 and L4-5 levels (Fig. 1). The lesion at L4-5 was oval with an isointense signal on T1-weighted images and low-signal intensity on T2-weighted images. The lesion at T12-L1 was semi-oval with an isointense signal on T1-weighted images and lowto high-signal inhomogeneous intensity on T2-weighted images. Surgical treatment was planned based on suspicion of benign multiple schwannoma and ependymoma, but MRI taken 3 weeks after the first visit showed growth and an increased number of intradural tumors. Gadolinium-contrast
T1-weighted images showed diffuse enhancement in the tumorous lesions (Fig. 1).

The rapid changes on MRI led us to strongly suspect a malignant tumor, and we performed a whole-body imaging test. MRI of the cervical and thoracic spinal cord showed enhanced skip lesions on contrast-enhanced T1-weighted images, but brain MRI showed no brain metastases. Computed tomography (CT) of the chest, abdomen and pelvis, and whole-body positron emission tomography (PET) showed no disease (Fig. 2). From these findings, a malignant tumor was suspected, including metastases of meningeal dissemination, malignant peripheral nerve sheath tumor, malignant lymphoma, and anaplastic ependymoma (WHO grade 3).

The tumor occupied the entire space within the dural tube at the T12-L1 level on a transaxial MR image, and the right leg-dominant neurological deficit had rapidly progressed. For diagnosis and prevention of neurological deficits, laminectomy between $\mathrm{T} 12$ and $\mathrm{L} 1$ and tumor resection were performed. The amplitude of intraoperative neurologic monitoring (motor-evoked potentials: MEPs) in the right quadriceps, tibialis anterior, gastrocnemius muscles and sphincter dropped during tumor detachment, and gross total resection was difficult due to severe adhesion to the cauda equina and epiconus. 

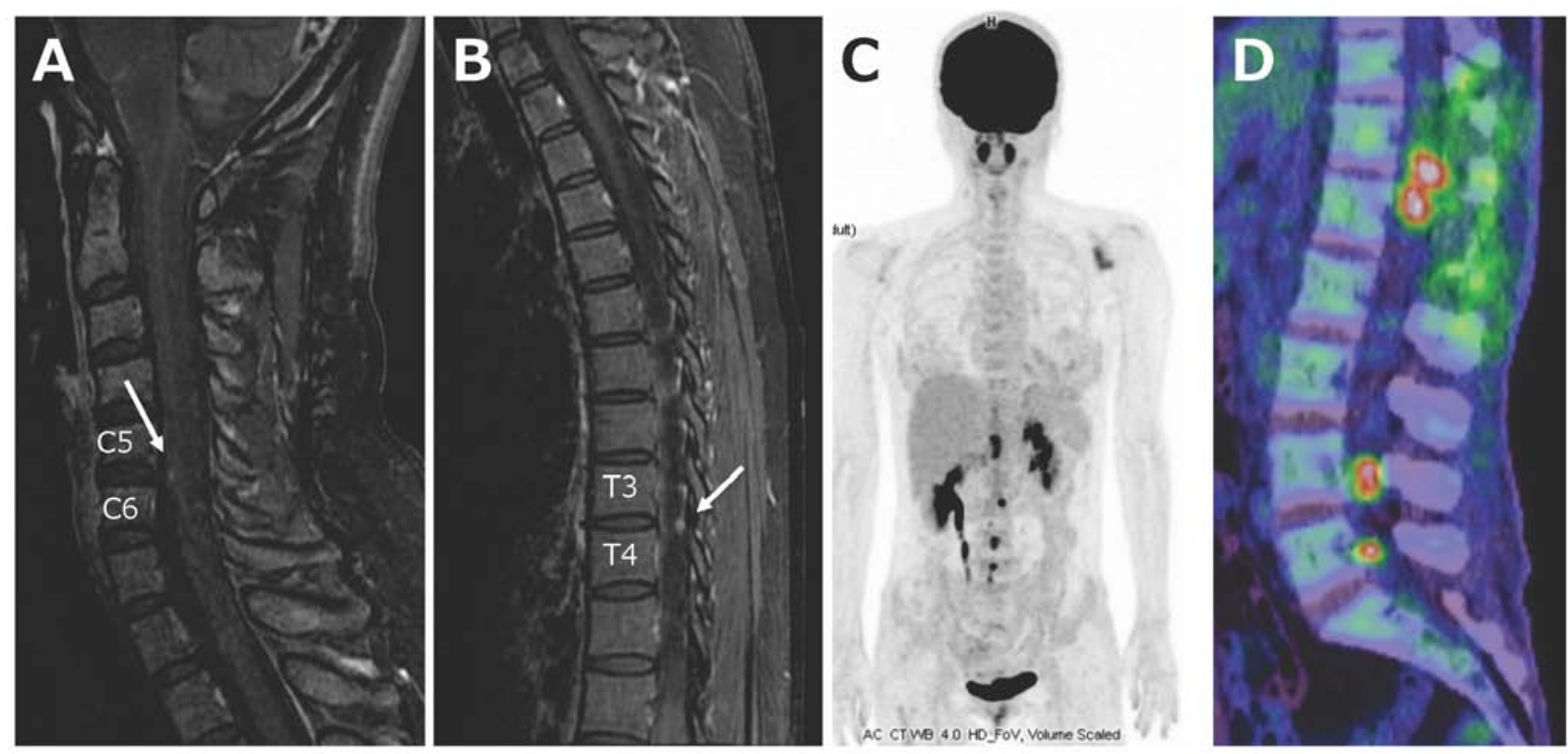

Figure 2. Gadolinium-contrast sagittal T1-weighted images of the (A) cervical and (B) thoracic spinal cord revealed meningeal seeding at C5-6 and T3-4 (arrows). (C) Whole-body PET was negative for disease other than that at the thoracolumbar level. (D) Multiple nodules were positive at the lumbar level in fluorodeoxyglucose-PET. PET, positron emission tomography.

A

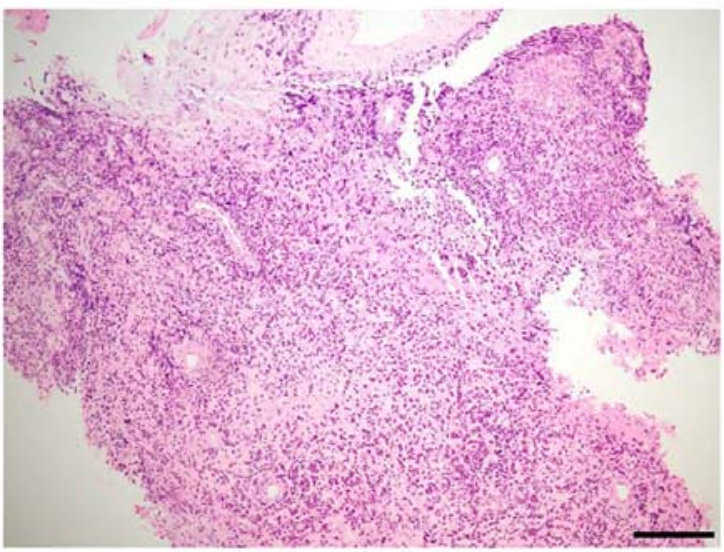

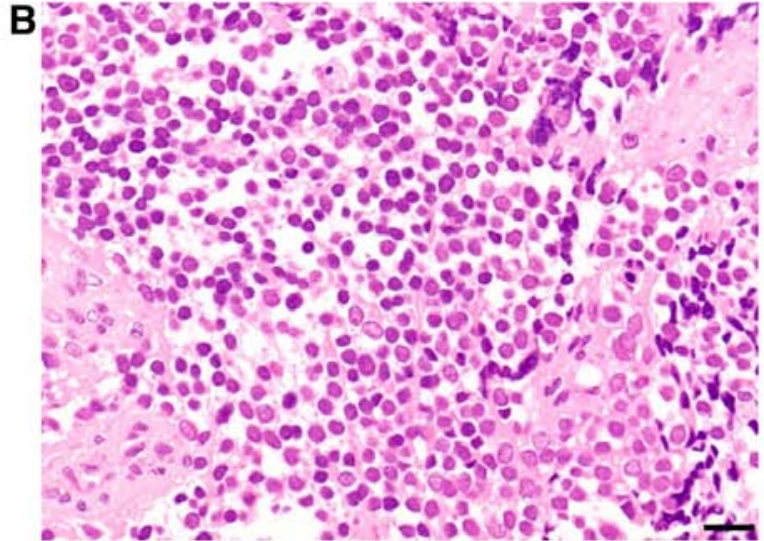

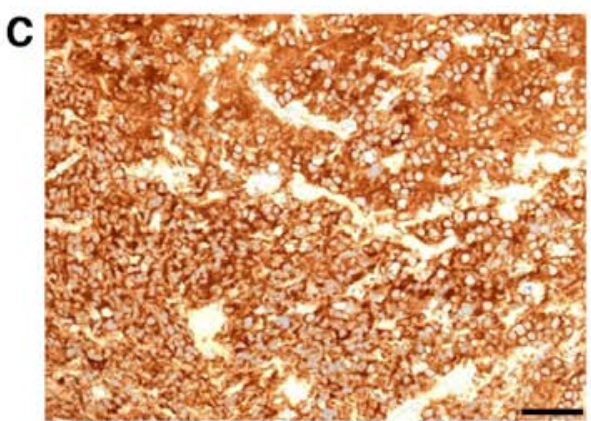

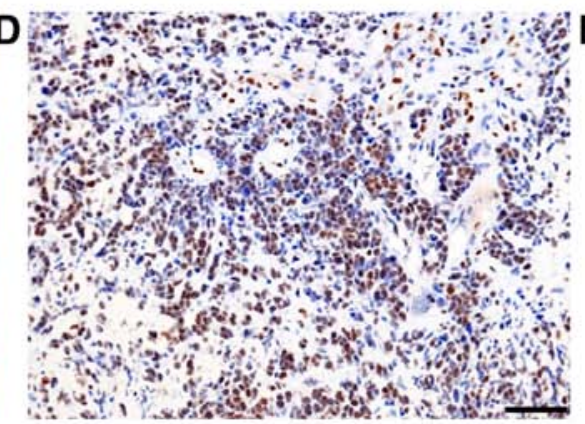

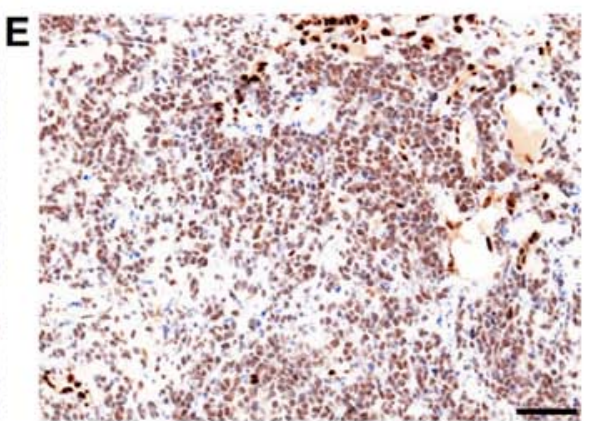

Figure 3. Photomicrographs of resected samples. (A) Low-power and (B) high-power images of haematoxylin and eosin staining. Immunohistochemical findings revealed positive staining for (C) CD99, (D) Fli-1 proto-oncogene, ETS transcription factor and (E) ETS transcription factor ERG. Scale bars, $200 \mu \mathrm{m}$ (A), $20 \mu \mathrm{m}$ (B) and $50 \mu \mathrm{m}$ (C-E).

Microscopically, the tumor consisted of dense sheets of small round cells based on hematoxylin-eosin staining. Immunohistochemically, most tumor cells showed intense and diffuse staining for CD99, ERG and FLI1, and were negative for GFAP, EMA, AE1/AE3, S-100, chromogranin A, and CD57 (Fig. 3). A nucleotide sequence analysis of the reverse transcription-polymerase chain reaction (RT-PCR) products confirmed that the gene fusion was formed between exon 7 of the EWSR1 gene and exon 5 of the FLI1 gene (Fig. 4). The lesion was diagnosed as primary intradural Ewing sarcoma at the cauda equina with meningeal seeding. 


\section{EWSR1-FLi1 fusion gene}

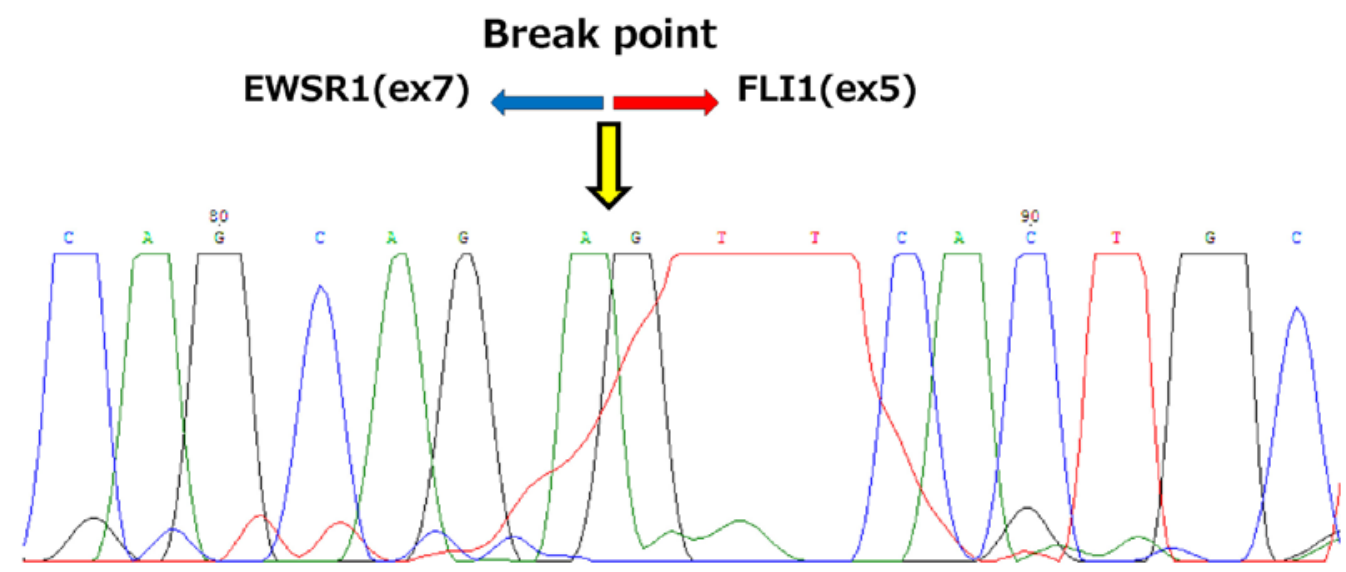

Figure 4. Partial nucleotide sequences containing junction of the fusion transcripts in the case. A nucleotide sequence analysis of the reverse transcription-PCR products demonstrated that the gene fusion was formed between exon 7 of the EWSR1 gene and exon 5 of the FLI1 gene. FLI1, Fli-1 proto-oncogene, ETS transcription factor; EWSR1, EWS RNA binding protein 1.

A

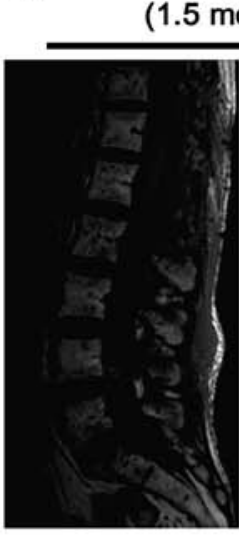

After radiation

onths after diagnosis)

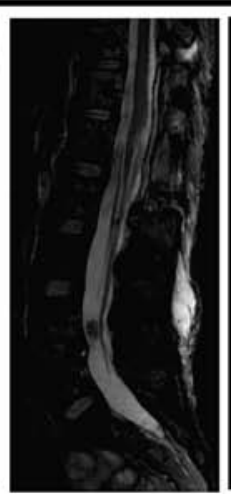

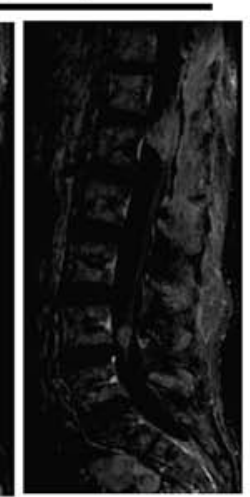

B

After chemotherapy (9 months after diagnosis)
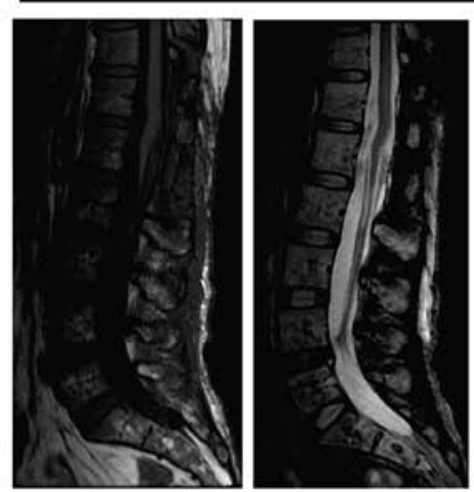

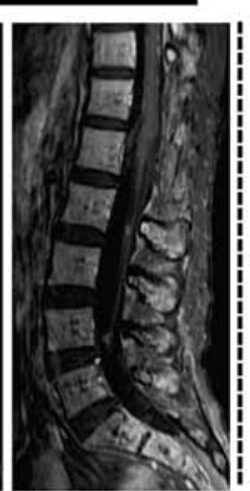

C 10 months after diagnosis

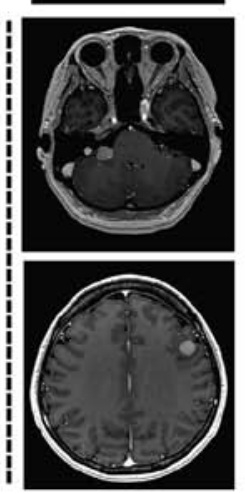

Figure 5. Sagittal thoracolumbar MRI revealing tumor shrinkage (A) after radiotherapy (1.5 months after diagnosis) and (B) chemotherapy (9 months after diagnosis). (C) Axial brain MRI revealing multiple brain metastases at 10 months after diagnosis.

Chemotherapy $(8$ cycles of vincristine, doxorubicin and cyclophosphamide (VDC) alternating with ifosfamide and etoposide (IE)) following radiotherapy (total dose of $45 \mathrm{~Gy}$ in 25 fractions for the whole spinal lesion excluding the brain) were performed after diagnosis. For Ewing sarcoma, we usually administer vincristine $\left(1.5 \mathrm{mg} / \mathrm{m}^{2}\right.$ on day 1$)$, doxorubicin $\left(37.5 \mathrm{mg} / \mathrm{m}^{2}\right.$ on days 1 and 2), and cyclophosphamide $\left(1200 \mathrm{mg} / \mathrm{m}^{2}\right.$ on day 1 ) (VDC) alternating with ifosfamide $\left(1.8 \mathrm{~g} / \mathrm{m}^{2}\right.$ on days $\left.1-5\right)$ and etoposide $\left(100 \mathrm{mg} / \mathrm{m}^{2}\right.$ on days $\left.1-5\right)$ (IE) every 3 weeks. The first course of VDC and second course of IE were administered as full doses. However, we reduced the dosage to $75 \%$ and delayed the start of chemotherapy for 1 week in the 3rd to 6th courses due to severe myelosuppression, and we reduced the dosages to $50 \%$ of standard chemotherapy in the 7th and 8th courses. Neurological symptoms improved with tumor shrinkage after radiation and chemotherapy; however, multiple brain metastases presenting with symptoms of double vision and hoarseness were found at 10 months after diagnosis (Fig. 5). Whole-brain irradiation and chemotherapy with another regimen were administered, but the patient died of diffusely disseminated disease limited to the central nervous system, without evidence of distant metastases, at 16 months after the initial diagnosis.

Histopathology. Histopathological examination was performed on resected specimen fixed $10 \%$ formalin for 24 hours at room temperature. Paraffin-embedded sections cut by $4 \mu \mathrm{m}$ thickness were performed hematoxylin and eosin (H\&E) and following immunohistological staining. For immunohistological examination, we used CD99 antibody (cat. no. M3601, DAKO) (1:300 dilution), FLI1 antibody (cat. no. ab15289, abcam) (1:50 dilution) and ERG antibody (cat. no. 41811, Nichirei). For CD99 immunostaining, we didn't perform antigen activation after deparaffinization. For FLI1 immunostaining, we performed antigen activation at $95^{\circ} \mathrm{C}$ for 20 minutes at $\mathrm{pH} 6.0$ using citrate buffer. For ERG immunostaining, we performed antigen activation at $95^{\circ} \mathrm{C}$ for 20 minutes at $\mathrm{pH} 9.0$ using TE buffer. We adopted the protocol by using the BONDIII Fully Automated IHC and ISH Stainer (Leica Microsysems, Japan) for all immunohistological staining. Slides were observed under a light microscope. 
RT-PCR and sequencing analyses. Total RNA (53 ng/ $\mu \mathrm{l})$ was extracted from FFPE sections using ISOGEN (Nippon Gene), according to the manufacturer's instructions. For specific amplification of the putative EWSR1-FLI1 junction regions, we performed a two-step PCR reaction. The sequences of first primers are EWSR1(ex6) forward, GAGACTAGTCAACCTCAATCTAGC and FLI1(ex6) reverse, AAGCTCCTCTTCTGACTGTG, EWSR1(ex7s) forward CCCACTAGTTACCCACCCCAAA and FLI1(ex8) reverse, GCCCAGGATCTGATACGGAT. The first PCR products were used as template for subsequent PCR reaction using the following nest primers; EWSR1(ex7s) forward, CCCACTAGTTACCCACCCCAAA and FLI1(ex5) reverse, TCGGTGTGGGAGGTTGTATT and FLI1(ex7) reverse, TGATCGTTTGTGCCCCTCCA. The first and nested PCR were performed with the following cycling conditions: $94^{\circ} \mathrm{C}$ for $3 \mathrm{~min}, 40$ cycles of $94^{\circ} \mathrm{C}, 60^{\circ} \mathrm{C}$, and $72^{\circ} \mathrm{C}$ for $1 \mathrm{~min}, 72^{\circ} \mathrm{C}$ for $10 \mathrm{~min}$. ABI PRIZM BigDye Terminator Cycle Sequencing Ready Reaction kits (PE Biosystems) was used for direct sequencing of the nested PCR products. EWSR1-FLI1 fusion gene analysis was performed using ABI PRISM 310 Genetic Analyzer (PE Biosystems). Sequencing reaction products were electrophoresed on $2 \%$ agarose gels and stained with ethidium bromide.

Statistical analysis. The statistical analysis was conducted using SPSS software (v24.0, SPSS). The Kaplan-Meier method was used for calculation of survival. Overall survival (OS) was defined as the time from diagnosis until the most recent follow-up or death of any cause. Progression-free survival was defined as the time from diagnosis until disease progression, death of any cause, or most recent follow-up.

\section{Discussion}

We present an extremely rare case of primary intradural extramedurally Ewing sarcoma (IEES). Clinical information for 30 cases of primary IEES reported from 1997 to 2019 is summarized in Table I (6-30). Of the 30 patients, 18 were male (60\%) and 12 were female (40\%). The median age at diagnosis was 31 years. The lumbar-sacral region was the most common location $(n=20,66.7 \%)$, and multiple lesions viewed as meningeal dissemination were found in 8 patients (26.7\%) at diagnosis. The most common chief symptom was pain $(n=25,83.3 \%)$. Motor disturbance of a lower or upper limb occurred in 15 patients $(50.0 \%)$, and bladder and rectal disturbance were present in 8 patients (26.7\%).

Ewing sarcoma is categorized as a small round cell sarcoma with pathognomonic molecular findings and varying degrees of neuroectodermal differentiation by immunohistochemistry. Classic Ewing sarcoma lacks neural differentiation and typically has only characteristic diffuse membranous CD99 (encoded by the MIC gene) positivity (31). Almost all cases in Table I $(n=28,93.3 \%)$ showed CD99 or MIC2 positivity. Ewing sarcoma has a specific translocation involving the EWSR1 gene on chromosome 22, which produces an EWSR1-FLI1 fusion gene transcript and oncoprotein. RT-PCR or fluorescence in situ hybridization (FISH) can be used to detect the fusion gene, and this was detected in 16 of the reported primary IEES cases. The 1- and 5-year OS rates

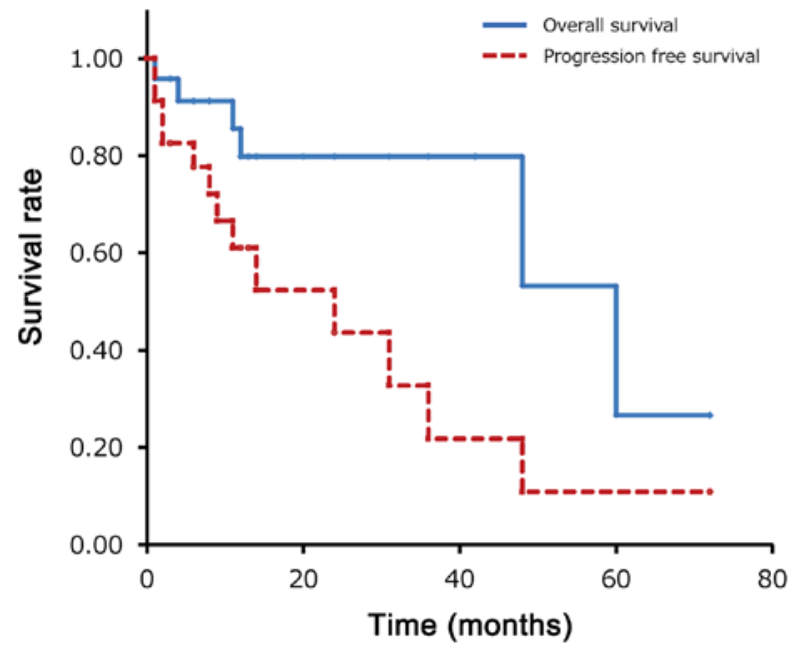

Figure 6. Kaplan-Meier curves for progression-free survival and overall survival. The 1- and 5-year overall survival rates were 79.8 and $26.6 \%$, respectively. The 1-, 2- and 5-year progression-free survival rates were 61.0, 52.3 and $10.9 \%$, respectively.

were $79.8 \%$ and $26.6 \%$, and the 1-, 2- and 5-year progression free survival rates were $61.0 \%, 52.3 \%$ and $10.9 \%$ (Fig. 6). Although the Kaplan-Meier survival analysis has certain limitation because the patients in Table I had a short duration of follow-up, these results suggest that IEES has a poorer prognosis than conventional localized Ewing sarcoma, which has a 5 -year OS of $65-75 \%$. The prognosis of IEES was the same as that of conventional Ewing sarcoma with metastases (4).

In general, patients with Ewing sarcoma receive neoadjuvant chemotherapy upon diagnosis of Ewing sarcoma by biopsy. However, spinal tumors are usually diagnosed after resection. All patients in Table I also received adjuvant chemotherapy after diagnosis. In the current case, multiagent chemotherapy was given, including VDC alternating with IE, as described above. However, we had to reduce the dosage and delay the start of chemotherapy for 1 week after the 3rd course because of severe myelosuppression. Zhang et al (32), suggested that adults with Ewing sarcoma should be treated with adequate cycles of intensive chemotherapy at appropriate intervals. Therefore, it is possible that the effect of chemotherapy was not sufficient in our case.

Ewing sarcoma is radiosensitive (33), and in our case we used whole spine radiotherapy without whole brain irradiation. Chihak et al (26), suggested that craniospinal radiotherapy, rather than focal radiotherapy, is critical for preventing distant metastasis, and skip metastases of intradural Ewing sarcoma to distant sites have been reported $(11,20,26)$. The patient in our case had a 2-year-old baby, and she refused craniospinal radiotherapy because she was afraid of late cerebral dysfunction. The outcome in this case suggests that craniospinal radiotherapy might be more effective for local control than whole spine radiotherapy.

In conclusion, IEES is an extremely rare malignant tumor that requires multimodal therapy with surgery, craniospinal radiotherapy and systematic chemotherapy. The poorer prognosis of primary IEES compared to that of conventional Ewing sarcoma of bone suggests that new agents and treatment strategies are needed. 


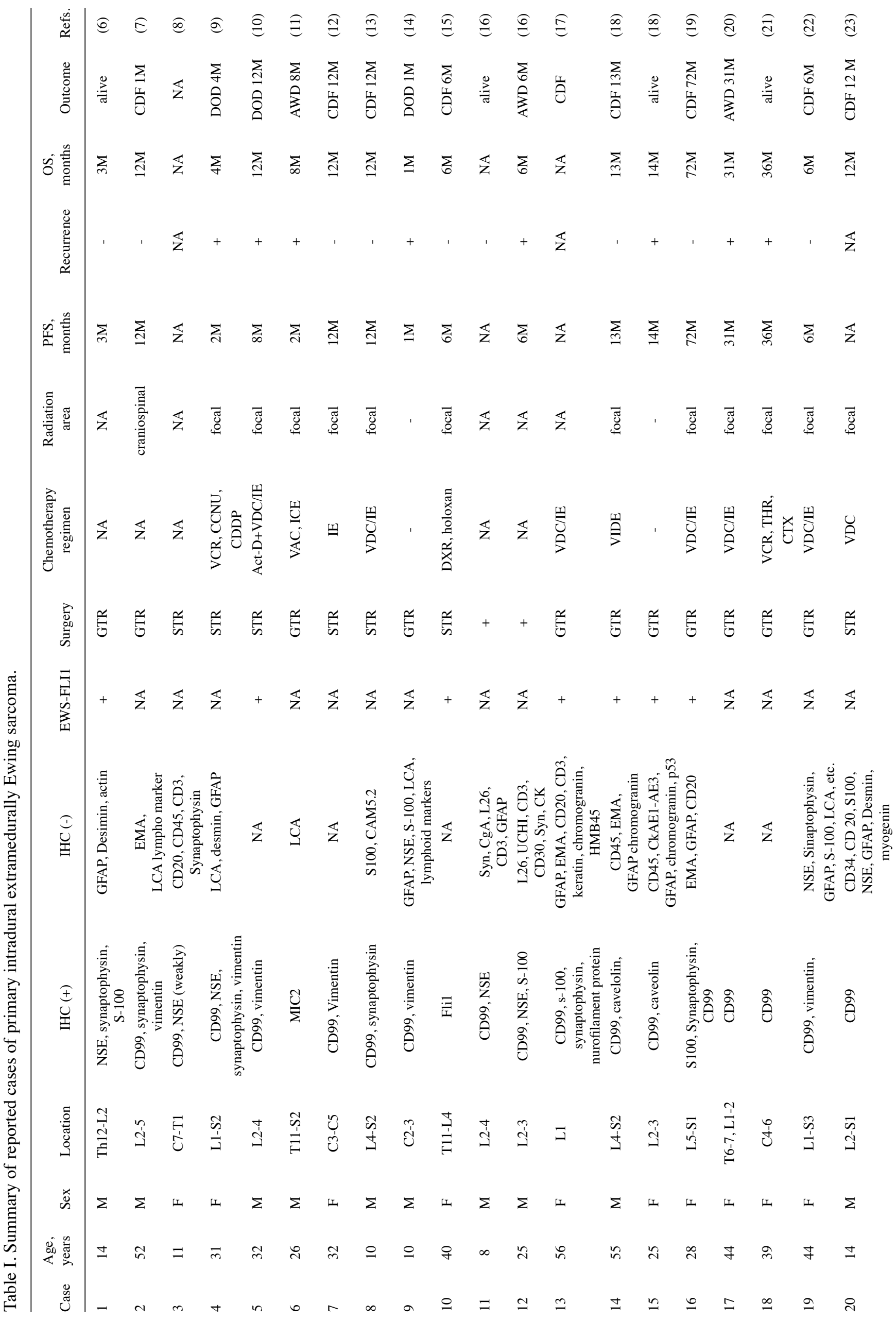




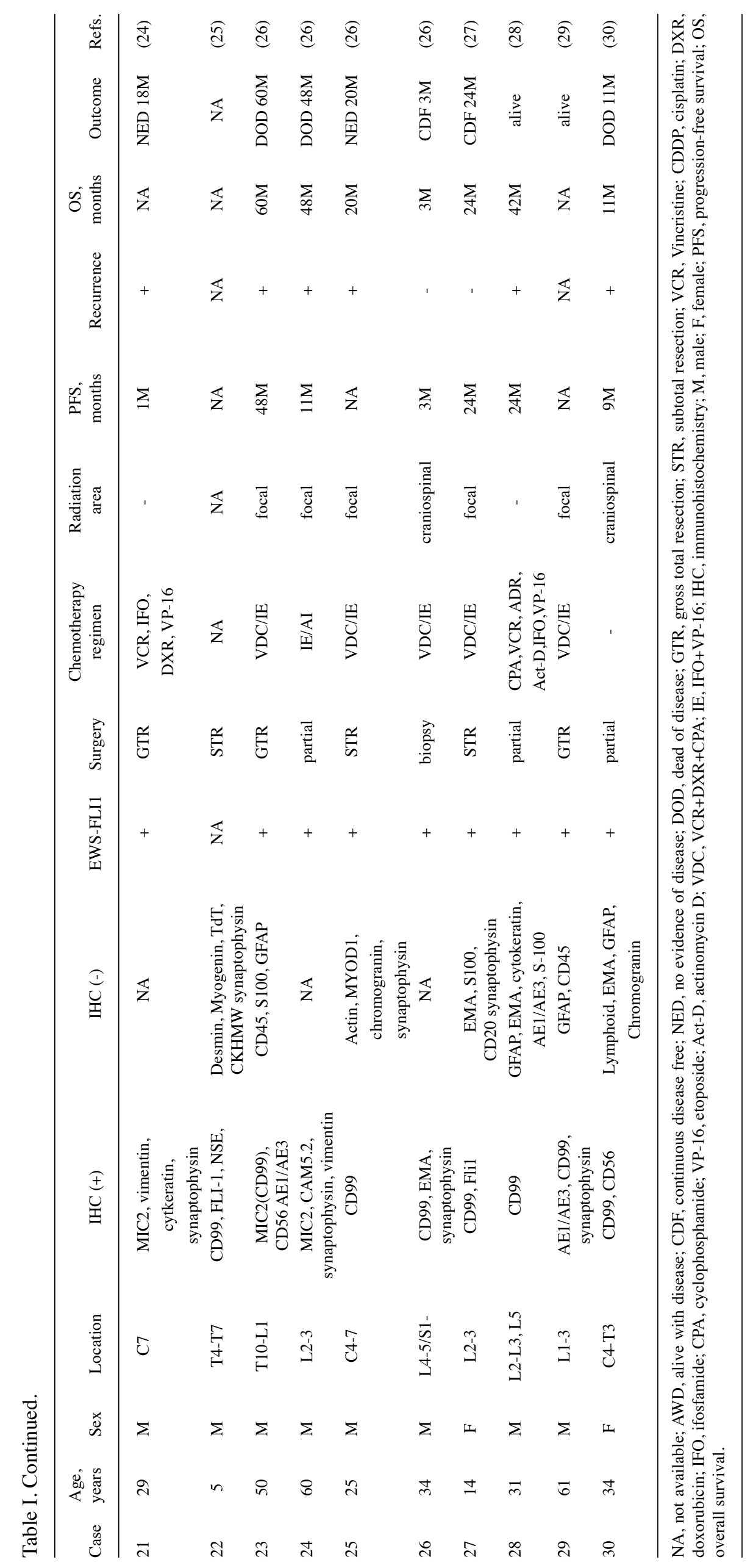




\section{Acknowledgements}

The authors would like to acknowledge Mr. Manabu Yamashita, laboratory technician at the Department of Pathology, Kanazawa University, Kanazawa, Japan, for performing of FISH and PCR tests.

\section{Funding}

No funding was received.

\section{Availability of data and materials}

All data generated or analyzed during this study are included in this published article.

\section{Authors' contributions}

YIz and $\mathrm{HN}$ examined the patient, analyzed the clinical, radiological and laboratory results, and wrote the manuscript. $\mathrm{KH}$ assisted with data analysis. $\mathrm{HN}$ and $\mathrm{KH}$ were involved in the surgical treatment. YIm and TN analyzed and interpreted the pathological findings and FISH tests. AM designed the study, including proofreading of the manuscript and revising it critically. $\mathrm{HN}$ and AM proofread the manuscript and revised it critically. AM made critical revisions of the article for important intellectual content. All authors read and approved the final manuscript.

\section{Ethics approval and consent to participate}

The study protocol was approved by the Human Ethics Review Committee of Fukui University Medical Faculty (Histological and biological analysis of disorders affecting the spine, bones and joints; approval no. 2014046) and strictly followed the Clinical Research Guidelines of the Ministry of Health, Labor, and Welfare of the Japanese Government.

\section{Patient consent for publication}

The patient and parents provided written informed consent for analysis and publication of the case.

\section{Competing interests}

The authors declare that they have no competing interests.

\section{References}

1. Van Goethem JW, van den Hauwe L, Ozsarlak O, De Schepper AM and Parizel PM: Spinal tumors. Eur J Radiol 50: 159-176, 2004.

2. Harimaya K, Oda Y, Matsuda S, Tanaka K, Chuman H and Iwamoto Y: Primitive neuroectodermal tumor and extraskeletal Ewing sarcoma arising primarily around the spinal column: Report of four cases and a review of the literature. Spine 28: E408-E412, 2003.

3. Balamuth NJ and Womer RB: Ewing's sarcoma. Lancet Oncol 11: 184-192, 2010

4. Gaspar N, Hawkins DS, Dirksen U, Lewis IJ, Ferrari S, Le Deley MC, Kovar H, Grimer R, Whelan J, Claude L, et al: Ewing sarcoma: Current management and future approaches through collaboration. J Clin Oncol 33: 3036-3046, 2015.

5. Applebaum MA, Worch J, Matthay KK, Goldsby R, Neuhaus J, West DC and Dubois SG: Clinical features and outcomes in patients with extraskeletal Ewing sarcoma. Cancer 117: 3027-3032, 2011.
6. Hisaoka M, Hashimoto $\mathrm{H}$ and Murao T: Peripheral primitive neuroectodermal tumour with ganglioneuroma-like areas arising in the cauda equina. Virchows Arch 431: 365-369, 1997.

7. Isotalo PA, Agbi C, Davidson B, Girard A, Verma S and Robertson SJ: Primary primitive neuroectodermal tumor of the cauda equina. Hum Pathol 31: 999-1001, 200. https://doi. org/10.1053/hupa.2000.16532.

8. Uesaka T, Amano T, Inamura T, Ikezaki K, Inoha S, Takamatsu M, Iwaki T and Fukui M: Intradural, extramedullary spinal Ewing's sarcoma in childhood. J Clin Neurosci 10: 122-125, 2003.

9. Akyüz M, Demiral AN, Gürer IE, Uçar T and Tuncer R: Primary primitive neuro-ectodermal tumor of cauda equina with intracranial seeding. Acta Neurochir (Wien) 146: 525-528, 2004.

10. Mobley BC, Roulston D, Shah GV, Bijwaard KE and McKeever PE: Peripheral primitive neuroectodermal tumor/Ewing's sarcoma of the craniospinal vault: Case reports and review. Hum Pathol 37: 845-853, 2006.

11. Haresh KP, Chinikkatti SK, Prabhakar R, Rishi A, Rath GK, Sharma DN and Julka PK: A rare case of intradural extramedullary Ewing's sarcoma with skip metastasis in the spine. Spinal Cord 46: 582-584, 2008

12. Kim SW and Shin H: Primary intradural extraosseous Ewing's sarcoma. J Korean Neurosurg Soc 45: 179-181, 2009.

13. Klimo P Jr, Codd PJ, Grier H and Goumnerova LC: Primary pediatric intraspinal sarcomas. Report of 3 cases. J Neurosurg Pediatr 4: 222-229, 2009.

14. Yan $\mathrm{Y}, \mathrm{Xu} \mathrm{T}$, Chen J, Hu G and Lu Y: Intraspinal Ewing's sarcoma/primitive neuroectodermal tumors. J Clin Neurosci 18: 601-606, 2011.

15. Vincentelli F, Caruso G and Figarella-Branger D: Primary intradural Ewing's sarcoma of the cauda equina presenting with acute bleeding. Acta Neurochir (Wien) 152: 563-564, 2010.

16. Duan XH, Ban XH, Liu B, Zhong XM, Guo RM, Zhang F, Liang BL and Shen J: Intraspinal primitive neuroectodermal tumor: Imaging findings in six cases. Eur J Radiol 80: 426-431, 2011.

17. Karikari IO, Mehta AI, Nimjee S, Hodges TR, Tibaleka J, Montgomery C, Simpson L, Cummings TJ and Bagley CA: Primary intradural extraosseous Ewing sarcoma of the spine: Case report and literature review. Neurosurgery 69: E995-E999, 2011.

18. Pancucci G, Simal-Julian JA, Plaza-Ramirez E, García-Marcos R, Mayordomo-Aranda E and Botella-Asunción C: Primary extraosseous intradural spinal Ewing's sarcoma: Report of two cases. Acta Neurochir (Wien) 155: 1229-1234, 2013.

19. Khalatbari MR, Jalaeikhoo $H$ and Moharamzad Y: Primary intradural extraosseous Ewing's sarcoma of the lumbar spine presenting with acute bleeding. Br J Neurosurg 27: 840-841, 2013.

20. Bazzocchi A, Bacci A, Serchi E, Salerno A, Salizzoni E and Leonardi M: Intradural extramedullary Ewing's sarcoma. Recurrence with acute clinical presentation and literature review. Neuroradiol J 26: 476-481, 2013.

21. Gong HS, Huang QS, Liu GJ, Chen FH and Zhao HB: Cervical primary Ewing's sarcoma in intradural and extramedullary location and skip metastasis to Cauda Equina. Turk Neurosurg 25: 943-947, 2015.

22. Lozupone E, Martucci M, Rigante L, Gaudino S, Di Lella GM and Colosimo C: Magnetic resonance image findings of primary intradural Ewing sarcoma of the cauda equina: Case report and review of the literature. Spine J 14: e7-e11, 2014.

23. Zhao M, Zhang B, Liang F and Zhang J: Primary spinal intradural extraskeletal Ewing sarcoma mimicking a giant nerve sheath tumor: Case report and review of the literature. Int J Clin Exp Pathol 7: 9081-9085, 2014.

24. Bostelmann R, Leimert M, Steiger HJ, Gierga K and Petridis AK: The importance of surgery as part of multimodal therapy in rapid progressive primary extraosseous Ewing sarcoma of the cervical intra- and epidural space. Clin Pract 6: 897, 2016.

25. Kartal A and Akatli A: Primary intradural extraosseous Ewing's sarcoma in a young child. Childs Nerv Syst 32: 409-410, 2016.

26. Chihak MA, Ahmed SK, Lachance DH, Nageswara Rao AA and Laack NN: Patterns of failure and optimal radiotherapy target volumes in primary intradural extramedullary Ewing sarcoma. Acta Oncol 55: 1057-1061, 2016.

27. Scantland JT, Gondim MJ, Koivuniemi AS, Fulkerson DH and Shih CS: Primary spinal intradural extraosseous Ewing sarcoma in a pediatric patient: Case report and review of the literature. Pediatr Neurosurg 53: 222-228, 2018.

28. Paterakis K, Brotis A, Tasiou A, Kotoula V, Kapsalaki E and Vlychou M: Intradural extramedullary Ewing's sarcoma: A case report and review of the literature. Neurol Neurochir Pol 51: 106-110, 2017. 
29. Takami H, Kumar R, Brown DA and Krauss WE: Histologic features and prognosis of spinal intradural extramedullary ewing sarcoma: Case report, literature review, and analysis of prognosis. World Neurosurg 115: 448-452.e2, 2018.

30. Tan CH, Tan D, Phung TB and Lai LT: Primary intradural extramedullary Ewing sarcoma of the cervical spine: A case report and review of the literature. J Clin Neurosci 66: 280-284, 2019

31. Fletcher CDM, Bridge JA, Hogendoorn PCW and Mertens F (eds): WHO Classification of Tumours of Soft Tissue and Bone. 4th edition. IARC, Lyon, France, 2013.

32. Zhang J, Huang Y, Sun Y, He A, Zhou Y, Hu H, Yao Y and Shen $\mathrm{Z}$ : Impact of chemotherapy cycles and intervals on outcomes of nonspinal Ewing sarcoma in adults: A real-world experience. BMC Cancer 19: 1168, 2019.
33. El Weshi A, Allam A, Ajarim D, Al Dayel F, Pant R, Bazarbashi S and Memon M: Extraskeletal Ewing's sarcoma family of tumours in adults: Analysis of 57 patients from a single institution. Clin Oncol (R Coll Radiol) 22: 374-381, 2010 .

(i) $(-)$ This work is licensed under a Creative Commons Attribution-NonCommercial-NoDerivatives 4.0 International (CC BY-NC-ND 4.0) License. 